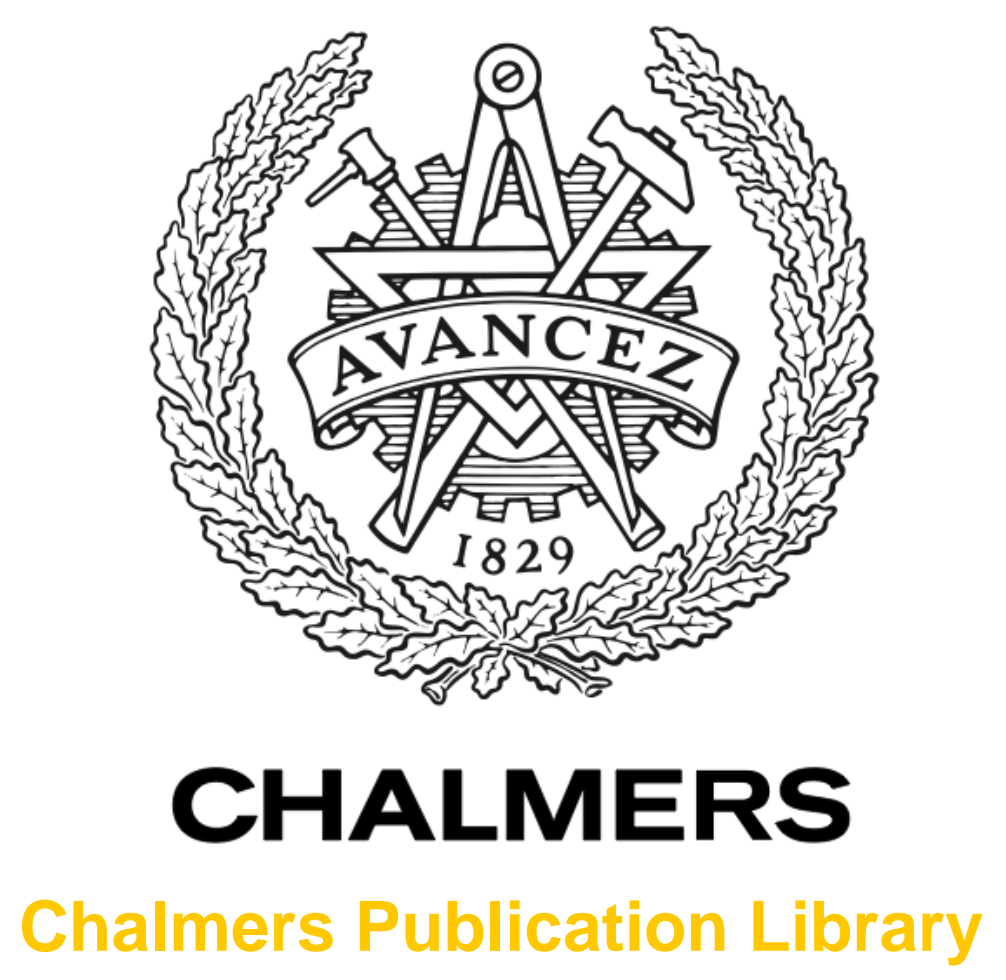

\title{
Optimization of the electron cooling by SIN tunnel junctions
}

This document has been downloaded from Chalmers Publication Library (CPL). It is the author's version of a work that was accepted for publication in:

\section{Superconductor Science and Technology}

Citation for the published paper:

Kuzmin, L. ; Agulo, I. ; Fominsky, M. (2004) "Optimization of the electron cooling by SIN tunnel junctions". Superconductor Science and Technology, vol. 17(5), pp. 400-405.

Downloaded from: http://publications.lib.chalmers.se/publication/327

Notice: Changes introduced as a result of publishing processes such as copy-editing and formatting may not be reflected in this document. For a definitive version of this work, please refer to the published source. Please note that access to the published version might require a subscription. 
Optimization of electron cooling by SIN tunnel junctions

This article has been downloaded from IOPscience. Please scroll down to see the full text article.

2004 Supercond. Sci. Technol. 17 S400

(http://iopscience.iop.org/0953-2048/17/5/062)

View the table of contents for this issue, or go to the journal homepage for more

Download details:

IP Address: 129.16.137.136

The article was downloaded on 05/07/2010 at 16:50

Please note that terms and conditions apply. 


\title{
Optimization of electron cooling by SIN tunnel junctions
}

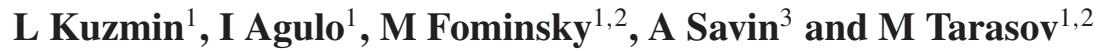 \\ ${ }^{1}$ Chalmers University, Institute of Microtechnology and Nanoscience, Gothenburg, Sweden \\ ${ }^{2}$ Institute of Radio Engineering and Electronics, 103907 Moscow, Russia \\ ${ }^{3}$ Low Temperature Laboratory, Helsinki University of Technology, PO Box 2200, \\ FIN-02015 HUT, Finland
}

E-mail: leonid.kuzmin@mc2.chalmers.se

Received 25 November 2003

Published 15 April 2004

Online at stacks.iop.org/SUST/17/S400

DOI: $10.1088 / 0953-2048 / 17 / 5 / 062$

\begin{abstract}
We report on the optimization of electron cooling by SIN tunnel junctions due to the advanced geometry of superconducting electrodes and very effective normal metal traps for more efficient removal of quasiparticles at temperatures from 25 to $500 \mathrm{mK}$. The maximum decrease in electron temperature of about $200 \mathrm{mK}$ has been observed at bath temperatures $300-350 \mathrm{mK}$. We used four-junction geometry with $\mathrm{Al}-\mathrm{AlO}_{x}-\mathrm{Cr} / \mathrm{Cu}$ tunnel junctions and $\mathrm{Au}$ traps. Efficient electron cooling was realized due to the improved geometry of the cooling tunnel junctions (quadrant shape of the superconducting electrode) and optimized Au traps just near the junctions $(\approx 0.5 \mu \mathrm{m})$ to reduce reabsorption of quasiparticles after removing them from normal metal. The maximum cooling effect was increased from a temperature drop of $\mathrm{d} T=-56 \mathrm{mK}$ (ordinary cross geometry) to $-130 \mathrm{mK}$ (improved geometry of superconducting electrodes) and to $\mathrm{d} T=-200 \mathrm{mK}$ (improved geometry of superconducting electrodes and effective Au traps).

The heating peak (instead of cooling) near the zero voltage across cooling junctions has been observed in practice for all samples at temperatures below $150 \mathrm{mK}$. For higher cooling voltages close to the superconducting gap, the heating was converted to cooling with decreased amplitude. The leakage resistance of the tunnel junctions gives a reasonable explanation of the heating peak. The phonon reabsorption due to the recombination of quasiparticles in superconducting electrodes gives an additional improvement in the theoretical fitting but could not explain the heating peak.

An anomalous zero-bias resistance peak has been observed for all tested structures. The peak is explained by Coulomb blockade of tunnelling in transistor-type structures with relatively small tunnel junctions.

The work on electron cooling is devoted to the development of a cold-electron bolometer (CEB) with capacitive coupling by SIN tunnel junctions to the antenna for sensitive detection in the terahertz region. Direct electron cooling of an absorber plays a crucial role in supersensitive detection in the presence of a realistic background power load.
\end{abstract}

(Some figures in this article are in colour only in the electronic version)

\section{Introduction}

In the last decade superconducting detectors have become the most sensitive radiation detectors of sub-millimetre, infrared and optical radiation with an estimated ultimate sensitivity down to $10^{-20} \mathrm{~W} \mathrm{~Hz}^{-1 / 2}$ [1]. Ultra-low-noise bolometers are required for space-based astronomical observations. The proposed NASA missions, SPIRE, SPIRIT and SPECS, will determine the highest level of requirements for bolometers for the near future. The detector goal is to provide a noise 
equivalent power of less than $10^{-20} \mathrm{~W} \mathrm{~Hz}^{-1 / 2}$ [2] over the 40 $500 \mu \mathrm{m}$ wavelength range in a $100 \times 100$ pixel detector array. No single existing technology could satisfy these requirements. The proposed concept of a cold-electron bolometer (CEB) with direct electron cooling [3,4] could be a good candidate to achieve the limit performance of the detector. The concept is based on the effect of nonequilibrium electron cooling by SIN tunnel junctions $[5,6]$. In contrast to cooling the membrane with a detector on it [7], we propose a CEB with very effective direct electron cooling of the absorber [3,4]. The CEB gives us the opportunity to remove incoming background power from the supersensitive region of the absorber to return the system to its lowest temperature state $\left(T_{\mathrm{e}}\right)$ to achieve the fundamental limit NEP [8]:

$$
\mathrm{NEP}^{2}=2 P_{0} k T_{\mathrm{e}} .
$$

For $P_{0}=10 \mathrm{fW}$ and $T_{\mathrm{e}}=50 \mathrm{mK}$ we would get the limit $\mathrm{NEP}=1 \times 10^{-19} \mathrm{~W} \mathrm{~Hz}^{-1 / 2}$.

The first step in this development is the realization of a strong electron cooling. This work has been devoted to the optimization of the superconducting electrodes and normal metal traps.

\section{Model}

The operation of a SINIS structure can be analysed using the heat balance equation $[9,10]$ :

$$
P_{\text {cool }}\left(V, T_{\mathrm{e}}, T_{\mathrm{ph}}\right)+\Sigma \Lambda\left(T_{\mathrm{e}}^{5}-T_{\mathrm{ph}}^{5}\right)=P_{0}+\frac{V^{2}}{R_{\mathrm{l}}}+\beta \frac{I}{e} \Delta+I^{2} R_{\mathrm{abs}} .
$$

Here, the cooling power is

$$
\begin{aligned}
& P_{\text {cool }}\left(V, T_{\mathrm{e}}, T_{\mathrm{ph}}\right) \\
& =\frac{1}{e^{2} R} \int \mathrm{d} E N_{\mathrm{S}}(E)(E-e V)\left[f_{\mathrm{N}}(E-e V)-f_{\mathrm{S}}(E)\right],
\end{aligned}
$$

$\Sigma \Lambda\left(T_{\mathrm{e}}^{5}-T_{\mathrm{ph}}^{5}\right)$ is the heat flow from the electron to the phonon subsystems in the normal metal, $\Sigma$ is a material constant, $\Lambda$ is the volume of the absorber, $T_{\mathrm{e}}$ and $T_{\mathrm{ph}}$ are, respectively, the electron and phonon temperatures of the absorber, $P_{\text {cool }}\left(V, T_{\mathrm{e}}, T_{\mathrm{ph}}\right)$ is the cooling power of the SIN tunnel junctions, $P_{0}$ is the background power load, $V^{2} / R_{1}$ is the power dissipation in the leakage resistance of the junction $R_{1}$ [11] and the third term accounts for the power flow back-tunnelling of quasiparticles and the reabsorption of phonons emitted by quasiparticle recombination [12]. The last term describes Joule heating of the absorber resistance and was usually small in comparison with the other terms. In simulations we have used the fifth- and sixth-order temperature dependence for heat flow between phonons and electrons. The energy diagram (figure 1(a)) illustrates the principle of electron cooling and the problem of reabsorption of the phonons emitted by quasiparticle recombination.

\section{Cooling experiments}

The first step of sample fabrication was evaporation of the normal metal traps. Au was chosen as the material for the traps fabricated in the same vacuum cycle as the contact pads. The pattern for the traps and the pads was formed
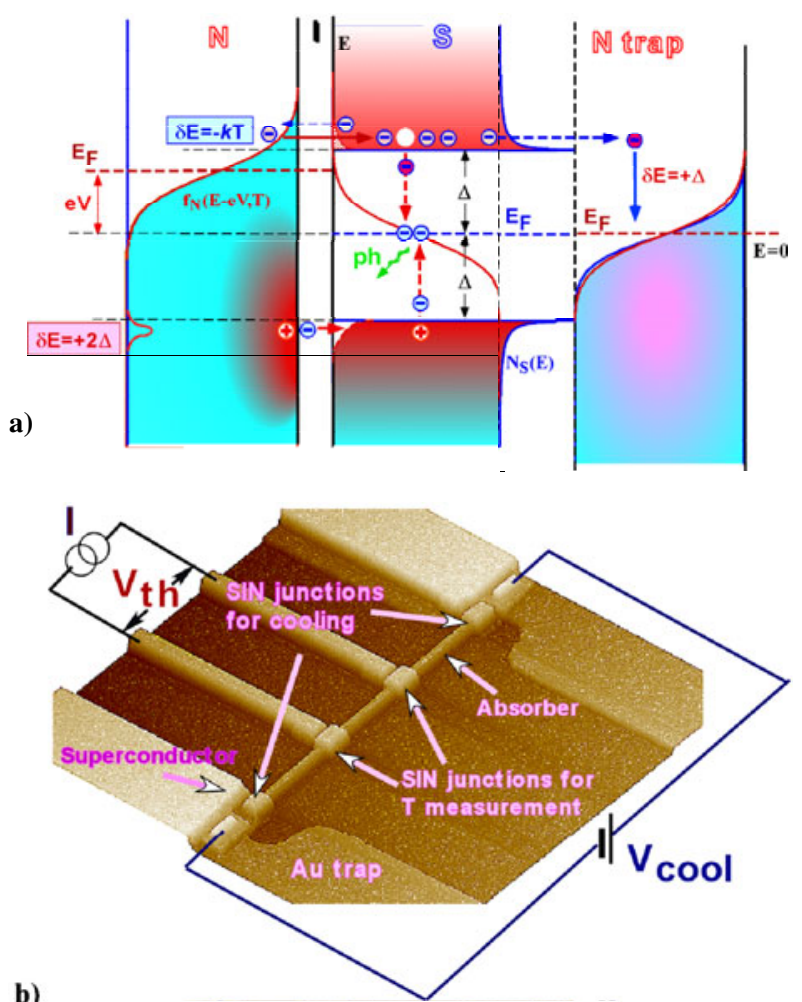

b)

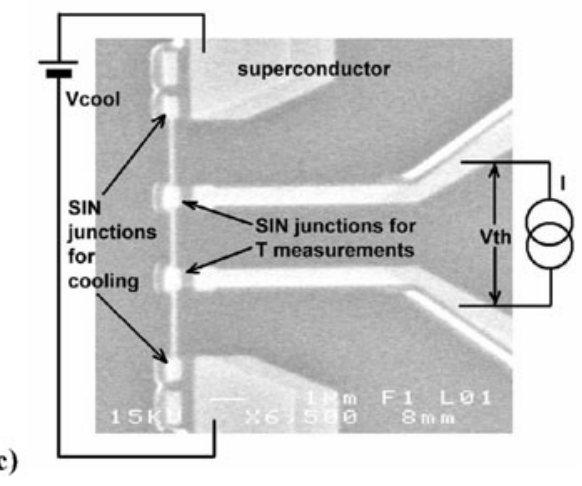

Figure 1. (a) Energy diagram illustrating the principle of electron cooling and the problem of reabsorption of the phonons after recombination of the quasiparticles. The SIN tunnel junction acts to cool the electrons by removing 'hot electrons' from the normal metal to the superconductor at a bias voltage lower than the gap voltage. A normal metal trap is placed in contact with the superconductor, allowing quasiparticles to be trapped and removed from the tunnel junction region. The removal of quasiparticles makes the cooling of the electrons in the normal metal more effective, decreasing the probability of back-tunnelling and phonon reabsorption. (b) AFM and (c) SEM images of the cooling structure made by the shadow evaporation technique. The Au trap (b) was evaporated prior to the evaporation of the $\mathrm{Al}-\mathrm{AlO}_{x}-\mathrm{Cr} / \mathrm{Cu}$ tunnel junctions.

using photolithography. Au was thermally evaporated up to a thickness of $600 \AA$. The next step was the fabrication of the tunnel junctions and the absorber. The structures were patterned by e-beam lithography and the metals were thermally evaporated using the shadow evaporation technique. The Al (superconductor) was evaporated at an angle of about $60^{\circ}$ up to a thickness of $650 \AA$ and oxidized at a pressure of $10^{-1} \mathrm{mbar}$ for $2 \mathrm{~min}$. A $\mathrm{Cr} / \mathrm{Cu}(1: 1)$ absorber with a total thickness of $750 \AA$ was then evaporated directly perpendicular to the substrate. 

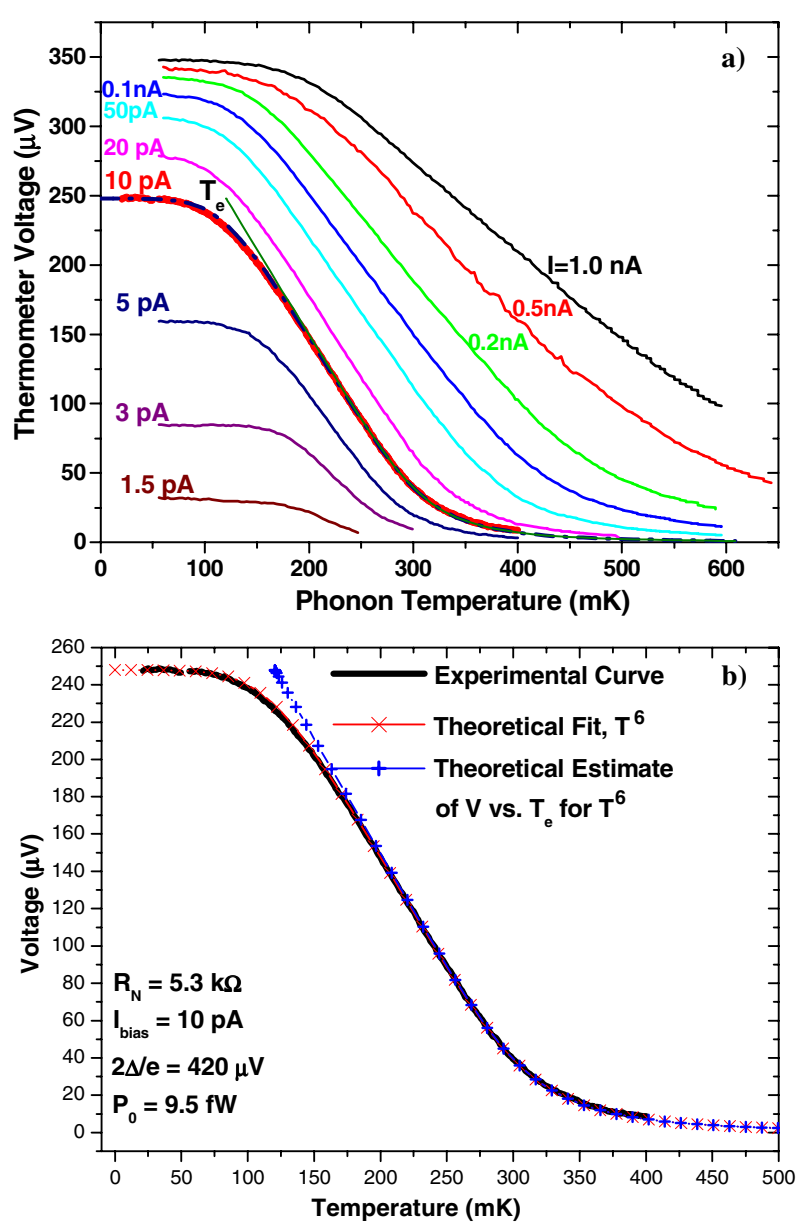

Figure 2. (a) Calibration of the thermometer junctions: the voltage is dependent on phonon temperature for different bias currents. (b) The theoretical fit and electron temperature are shown for the main calibration curve chosen for the cooling experiment with a bias current of $10 \mathrm{pA}$.

Figure 1(b) shows the configuration of the electrodes with an advanced geometry of the two outer (cooling) junctions and with normal metal traps. The cooling junctions have a normal state resistance $R_{\mathrm{N}}$ equal to $0.86 \mathrm{k} \Omega$, while the two thermometer junctions (in the central part of the absorber) have $R_{\mathrm{N}}$ equal to $5.3 \mathrm{k} \Omega$. The thermometer junctions have a simple cross geometry, where a section of the normal metal absorber overlaps the thin $\mathrm{Al}$ electrodes. The area of overlap, which is equivalent to the area of the tunnel junction, is equal to $0.2 \times 0.3 \mu \mathrm{m}^{2}$. The structure of the outer junctions is such that the ends of the normal metal absorber overlap a corner of each of the $\mathrm{Al}$ electrodes, which have a much larger area compared to the middle $\mathrm{Al}$ electrodes. The area of each of these junctions is $0.55 \times 0.8 \mu \mathrm{m}^{2}$. The purpose of the larger area Al electrode is to give more space for quasiparticle diffusion compared to the middle $\mathrm{Al}$ electrode with a simple cross geometry. In the structure described, the two cooler and thermometer junctions have a $R_{\mathrm{N}}$ equal to $0.85 \mathrm{k} \Omega$ and $5.4 \mathrm{k} \Omega$, respectively. The volume of the absorber is $0.18 \mu \mathrm{m}^{3}$ and its resistance is $120 \Omega$.

A bias cooling current is applied through the outer junctions and the absorber. These tunnel junctions act as the cooling junctions and therefore serve to decrease the electron temperature of the absorber. To determine the electron

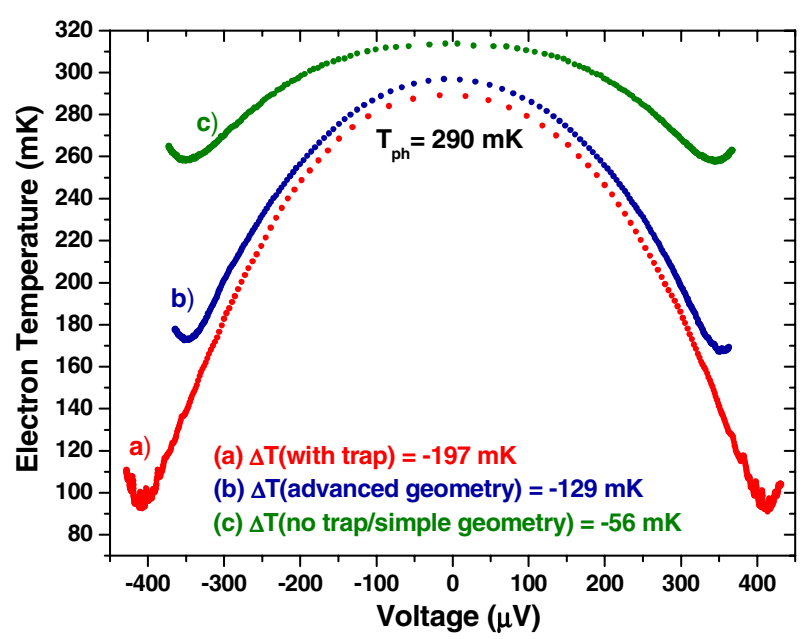

Figure 3. The main cooling curves: electron temperature of the absorber as a function of the voltage across the cooling SIN tunnel junctions for (a) the advanced geometry of the superconducting electrodes and effective Au traps, (b) the advanced geometry of the superconducting electrodes without traps, and (c) the ordinary cross geometry.

temperature, the voltage across the inner junctions is measured. This voltage is then calibrated as a function of temperature and is shown in figure 2 for the same bias current. The bias has to be optimal to obtain the maximum linear voltage response to temperature, and yet not too large so as to disturb the cooling process in the absorber.

For lower currents, it is limited by the leakage resistance of the tunnel junctions and for larger currents by the combination of overheating of the absorber by power dissipation in the leakage resistance of tunnel junctions and the beginning of the active cooling process (figure 2(a)). The optimal applied bias was $10 \mathrm{pA}$. Figure 2(b) shows the theoretical fit and electron temperature for the calibration curve with this bias current. The fit was obtained using equations (2) and (3) with a power load $9.5 \mathrm{fW}$ and leakage resistance $17 \mathrm{M} \Omega$ per junction.

Figure 3 shows the main results of electron cooling for different geometries of superconducting electrodes and effective Au traps. The experimental calibration curve of the measured voltage against temperature from figure 2(b) was used for conversion of the thermometer voltage to temperature. The electron temperature as a function of the cooling voltage is shown in figure 3 for various combinations of the geometry of superconducting electrodes and effective Au traps (a)-(c). A strong influence of the advanced geometry of superconducting electrodes (b) can be seen in comparison with the usual cross geometry (c). Back-tunnelling of quasiparticles and reabsorption of phonons contribute to the heating of the absorber [12]. With the advanced geometry, the probability of these processes is decreased due to the outdiffusion of quasiparticles from the junction area. The further improvement of cooling in comparison with (b) is obtained by the close position of effective Au traps (a) just near the tunnel junction $(\approx 0.5 \mu \mathrm{m})$. With the addition of normal metal traps (a), quasiparticles are allowed to dissipate their energies in the traps, thus further reducing the probability of quasiparticle back-tunnelling and quasiparticle recombination in the superconductor, enhancing the electron cooling process 

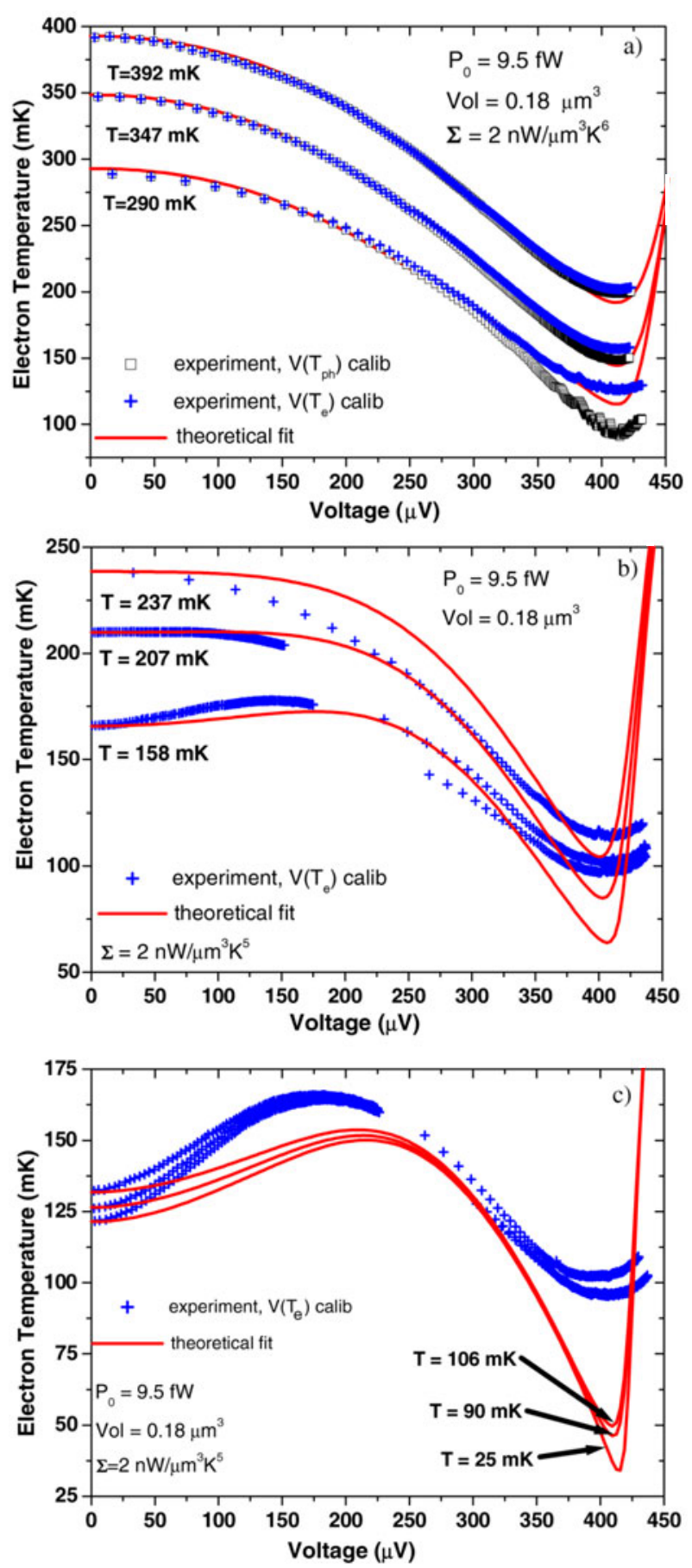

Figure 4. The main cooling curves and theoretical fit using a $T^{6}$ temperature dependence (a) and $T^{5}$ temperature dependence (b) and (c): electron temperature is shown as a function of the voltage across the cooling junctions for different temperature regions. The fitting parameters are shown on the graphs, the leakage resistance was taken from the experiment $R_{1}=1 \mathrm{M} \Omega$ and the reabsorption coefficient, $\beta=0.02$, was found from the fit.

of the absorber. Careful checking of $I-V$-curves shows the absence of visible suppression of the gap due to a proximity effect even for this close positioning of the normal metal traps.

Figure 4 shows the electron cooling curves and the theoretical fit for different temperatures for the structure with normal metal traps. In figure 4(a) we used for experimental curves both the experimental calibration curve of the measured

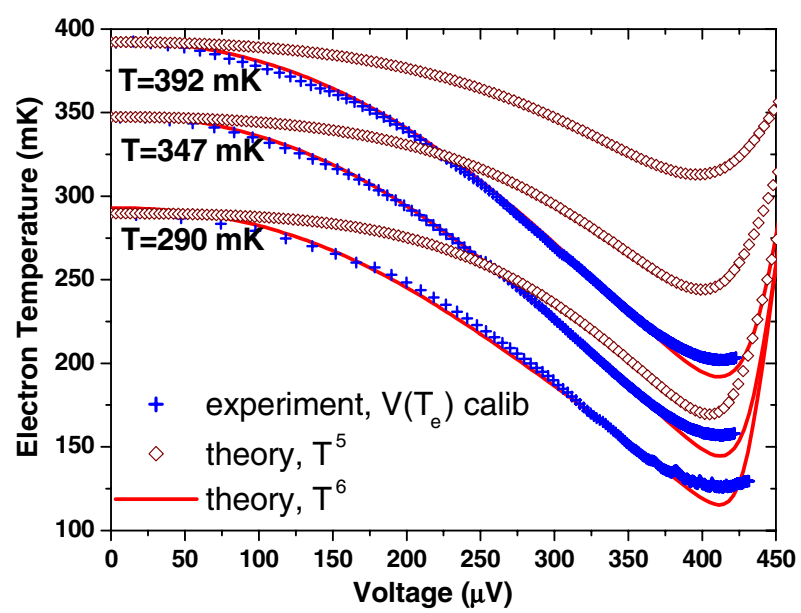

Figure 5. The main cooling curves and theoretical fit using $T^{5}$ and $T^{6}$ temperature dependences for comparison: electron temperature is shown as a function of the voltage across the cooling junctions for different temperature regions.

$V\left(T_{\mathrm{ph}}\right)$ from figure 2(b) and the theoretical estimation of $V\left(T_{\mathrm{e}}\right)$ shown in the same figure. For figures 4(b) and (c) we used only the calibration curve $V\left(T_{\mathrm{e}}\right)$, giving better coincidence with the theory.

The best coincidence of theoretical fit was obtained from equations (2) and (3) using the $T^{6}$ temperature dependence. The experimental results show very good agreement with theory for phonon temperatures in the range $300-400 \mathrm{mK}$ (figure 4(a)). The leakage resistance was taken from the experiment $R_{1}=1 \mathrm{M} \Omega$. The reabsorption coefficient, $\beta=$ 0.02 , and the material constant, $\Sigma=2 \mathrm{nW} \mu \mathrm{m}^{-3} \mathrm{~K}^{-6}$, was found from the fit. We speculate that the small discrepancy between theory and experiment around the optimal cooling voltage may be due to the stronger reabsorption of the quasiparticles from a superconducting electrode for maximum cooling. Overheating of the phonon system in the absorber should also be taken into account for more accurate analysis. The effect is stronger at low temperatures due to the lower thermal conductivity.

Initially, our theoretical calculations were made using the $T^{5}$ temperature dependence of heat flow between phonons and electrons. Comparison with experimental results (figure 5) showed that, as the voltage was increased towards the gap voltage, the electron temperature decreased faster than theory predicted. The electron cooling in the experiments was even better than in the theory. This suggests either a decoupling of the two phonon subsystems, i.e. the normal metal and substrate phonon subsystems, or a weaker coupling between electrons and phonons in the normal metal. Thus, either an additional decoupling or a higher degree of temperature dependence is needed to be introduced in the theory. The theory of disordered conductors $[13,14]$ predicts a higher power law for the electron-phonon scattering rate, $\tau_{\mathrm{e}-\mathrm{ph}} \propto T^{-4}$, that leads to a $T^{6}$ temperature dependence of the hot-electron effect. Therefore, in our simulations, we have used a $T^{6}$ temperature dependence in the upper temperature range for a better fit. The material constant, $\Sigma$, and the reabsorption coefficient, $\beta$, were used as fitting parameters. The reabsorption coefficients were approximately the same for all temperatures. On the other hand, the material constant has a dependence on temperature. 
Our calculations using the sixth power for temperature fit very nicely with our experimental data at high temperatures (>300 mK). A heating peak (instead of cooling) near the zero-cooling voltage has been observed at temperatures lower than $150 \mathrm{mK}$ in practice for all samples. At higher cooling voltages, close to the superconducting gap, the heating was converted to cooling with decreased amplitude. The power dissipation through the leakage resistance of the tunnel junctions (equation (2)) gives rather a good fitting for the heating peak. The phonon reabsorption due to the recombination of quasiparticles in superconducting electrodes gives an additional improvement of the theoretical fit but could not explain the heating peak without leakage resistance.

The problem of removing heat from the cooling junctions is the main limitation for the cooling operation. An accurate analysis of the thermal balance in the system, including the factors that contribute to this limitation, is rather complicated. However, we are aware of these factors. The efficiency of the tunnel junction as a cooler, i.e. the ratio between the total power applied to the cooler and the cooling power, is rather low and decreases with temperature. In addition, the thermal conductivity of the materials used (the substrate and $\mathrm{Au}$, for example) also has a temperature dependence. In the ideal case, most of the power should be absorbed by the phonon subsystem in the superconductor and the trap. This leads to temperature gradients between the normal metal and superconductor phonon subsystems, creating a thermal flow towards the normal metal. As the applied cooling voltage is increased towards the gap voltage, the temperature gradient also increases. As a result, the phonon temperature in the normal electrode is a function of the applied cooling voltage. All these factors contribute towards the overheating of the normal metal, affecting the cooling operation at lower temperatures.

\section{Anomalous zero-bias resistance peak}

In addition to the $V(T)$ calibration of the thermometer, we used the zero-bias resistance dependence $R_{0}(T)$ for electron temperature measurements. We determined $R_{0}$ of the thermometer junctions using a modulation technique with lock-in amplifier [15]. For the beginning, the $R_{\mathrm{d}}(V)$ has been measured (figure 6). From high voltages down to $150 \mu \mathrm{V}$, the curve shows a typical increase of the resistance due to an increase of the subgap resistance and, then, flattening due to leakage resistance $(17 \mathrm{M} \Omega$ ). Near the zero-bias voltage the curve shows an increase of the resistance, due to an anomalous zero-bias peak.

Unexpected behaviour of the anomalous zero-bias peak has been observed in its dependence on temperature. The $R_{0}(T)$ has been measured for both thermometer (9-13) and cooling (D10-12, L11-15) junctions (figure 7). The curves 10-12 and 11-15 show puzzling behaviours: an exponential increase from higher to lower temperatures down to $200 \mathrm{mK}$ and, then, a flattening, followed by an increase (!) near zero temperature. The curve for junction 9-13 shows an exponential increase of resistance from higher to lower temperatures, then an unpredictable change of slope to a linear dependence near $T=260 \mathrm{mK}$, and then flattening near $120 \mathrm{mK}$. Flattening can

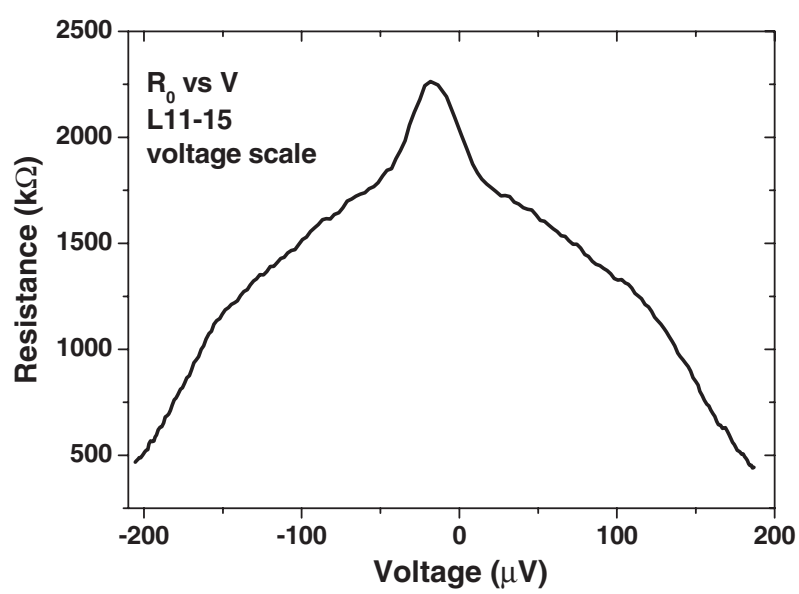

Figure 6. Voltage dependence of the differential resistance, $R_{\mathrm{d}}$, of the cooling junctions. The dependence shows some flattening due to leakage resistance $( \pm 150 \mu \mathrm{V})$ and, then, an anomalous zero-bias resistance peak at low voltages. This peak has been observed in practice for all structures The peak has been interpreted as a Coulomb blockade peak due to a SET transistor-type geometry of the cooling structure.

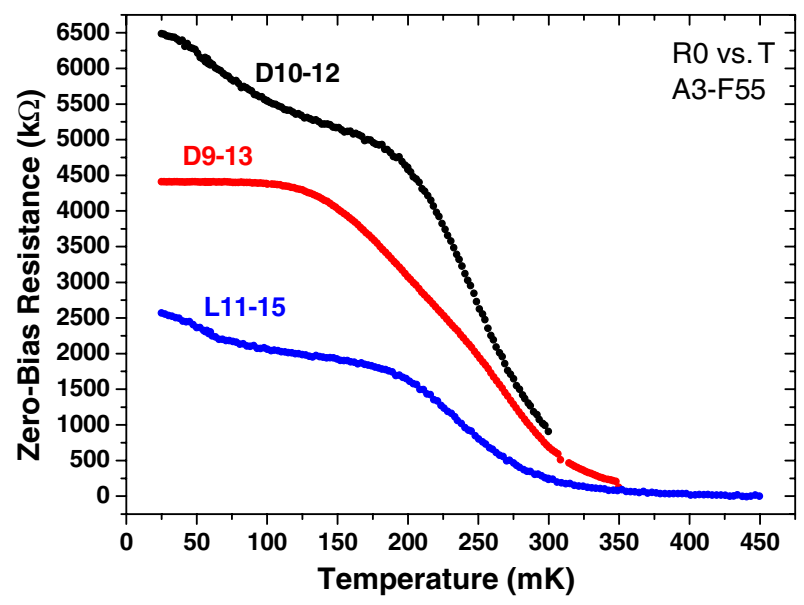

Figure 7. Temperature dependence of the zero-bias resistance, $R_{0}$, of the thermometer junctions (D9-13) and cooling junctions (D10-12 and L11-15). The resistance of D9-13 is scaled (decreased) 10 times for comparison with the other curves. The dependences show a flattening due to leakage resistance and an increase of the anomalous peak at low temperatures (different for smaller D9-13 junctions). The peak is interpreted as a Coulomb blockade peak due to a SET transistor-type geometry of the cooling structure.

be easily explained by leakage resistance but other features are rather puzzling.

The only explanation we can manage to find is due to Coulomb blockade of tunnelling in transistor-type structures with relatively small tunnel junctions. The estimation of typical parameters of the structures gives the figures shown in table 1 . Table 1 shows a rather good coincidence of the width of the peak measured in microvolts and converted to temperature, $T_{\text {peak }}$, and the width of peak decrease from figure $7, T_{\exp -V(T)}$, for structures 10-12 and 9-13. For structure L11-15 the coincidence is not as good, which is probably related to the accuracy of the peak measurements with modulation techniques. 
Table 1. Parameters of the SINIS cooling structure (junctions D9-13), two SIN thermometer junctions (D9-13) and the bolometer structure (L11-15). $V_{\mathrm{CB}}$ is the Coulomb blockade voltage determined from junction capacitance and absorber capacitance, $\Delta V_{\text {peak.exp }}$ is the experimental width of the peak, $T_{\text {peak }}$ is the temperature of the smearing proportional to $\Delta V_{\text {peak.exp }}$ and $T_{\exp -V(T)}$ is the experimental value of the smearing of the peak from figure 7.

\begin{tabular}{lllclcllc}
\hline Sample & $\begin{array}{l}\text { Area } \\
\left(\mu \mathrm{m}^{2}\right)\end{array}$ & $\begin{array}{l}R \\
(\mathrm{k} \Omega)\end{array}$ & $\begin{array}{l}R_{0} \\
(\mathrm{M} \Omega)\end{array}$ & $C(\mathrm{pF})$ & $\begin{array}{l}V_{\mathrm{CB}} \\
(\mu \mathrm{V})\end{array}$ & $\begin{array}{l}\Delta V_{\text {peak.exp }} \\
(\mu \mathrm{V})\end{array}$ & $\begin{array}{l}T_{\text {peak }} \\
(\mathrm{mK})\end{array}$ & $\begin{array}{l}T_{\exp -V(T)} \\
(\mathrm{mK})\end{array}$ \\
\hline $\mathrm{D} 10-12$ & 0.45 & 0.85 & 1.0 & 0.02 & 5.7 & 10 & 110 & 80 \\
$\mathrm{D} 9-13$ & 0.06 & 5.4 & 17 & 0.0018 & 44 & 20 & 220 & 260 \\
L11-15 & 0.45 & 0.9 & 1.4 & 0.02 & 5.7 & 22 & 260 & 80 \\
\hline
\end{tabular}

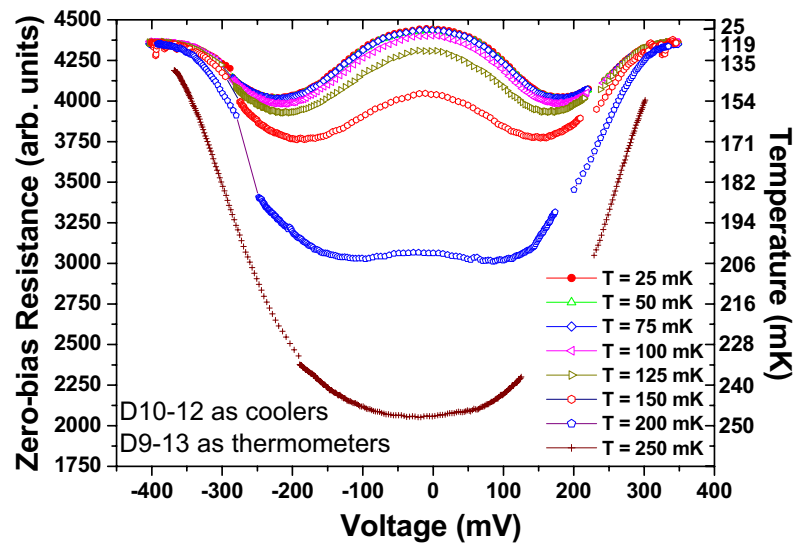

Figure 8. The zero-bias resistance, $R_{0}$, of the thermometer junctions (D9-13) and the electron temperature of the absorber as a function of the voltage across the cooling SIN tunnel junctions (D10-12) for various phonon temperatures. The experimental relation $R_{0}(T)$ from figure 7 was used for estimation of the electron temperature of the absorber.

The cooling dependences $R_{0}\left(V_{\text {cool }}\right)$ in figure 8 have been used for the estimation of the dependences $T_{\mathrm{e}}\left(V_{\text {cool }}\right)$ similar to those shown in figure 4 . The right axis shows these data converted to electron temperatures using the experimental calibration curve of figure 7 for thermometer junctions D913. Accuracy near the minimum temperature is rather low due to flattening of the calibration curve near the minimum temperature caused by leakage resistance. This reason reduces the accuracy of the temperature determination using $R_{0}(T)$ in comparison with $V(T)$. The Coulomb blockade peak causes additional difficulties in calibration and theoretical fitting for this calibration curve for the correct determination of the electron temperature.

\section{Conclusions}

The optimization of electron cooling by SIN tunnel junctions has been done for the advanced geometry of the superconducting electrodes (quadrant shape) and very effective Au traps just near the junctions $(\approx 0.5 \mu \mathrm{m})$ at temperatures from 25 to $500 \mathrm{mK}$. The maximum decrease in temperature of about $200 \mathrm{mK}$ has been observed for a combination of these two improvements.
The heating peak (instead of cooling) near the zerocooling voltage at temperatures lower than $150 \mathrm{mK}$ observed in practice for all samples, has been explained by the leakage resistance of the junctions. The phonon reabsorption due to the recombination of quasiparticles in superconducting electrodes gives an additional improvement in the theoretical fitting but could not explain on its own the heating peak.

The anomalous zero-bias resistance peak has been observed for all tested structures. The peak is explained by Coulomb blockade of tunnelling in a transistor-type structure with relatively small tunnel junctions.

\section{Acknowledgments}

The work was supported by the SI, VR and STINT Swedish agencies.

\section{References}

[1] Bitterman A 1999 Supercond. Cryoelectron. 1217

[2] Leisawitz D et al 2000 Scientific motivation and technology requirements for the SPIRIT and SPECS far-infrared/submillimeter space interferometers Proc. SPIE 401336

[3] Kuzmin L 2000 Physica B 284-288 2129

[4] Kuzmin L, Devyatov I and Golubev D 1998 Proc. SPIE 3465 193

[5] Nahum M and Martinis J M 1994 Appl. Phys. Lett. 653123

[6] Leivo M, Pecola J and Averin D 1996 Appl. Phys. Lett. 68 1996

[7] Pekola J et al 2000 Appl. Phys. Lett. 762782

[8] Kuzmin L 2003 Proc. ESA Workshop 'New Perspectives for Post-Herschel Far Infrared Astronomy from Space' (Madrid, Sept. 2003) http://damir.iem.csic.es/workshop/program.html

[9] Golubev D and Kuzmin L 2001 J. Appl. Phys. 896464

[10] Kuzmin L and Golubev D 2002 Physica C 372-376 378

[11] Savin A, Prunnila M, Ahopelto J, Kivinen P, Törmä P and Pekola J 2003 Application of superconductor-semiconductor Schottky barrier for electron cooling Physica B 329-333 1481-4

[12] Jochum J et al 1998 J. Appl. Phys. 833217

[13] Schmid A 1973 Z. Phys. 259421

Rammer J and Schmid A 1986 Phys. Rev. B 341352

[14] Reizer M Y and Sergeev A V 1986 Zh. Eksp. Teor. Fiz. 90 1056

Reizer M Y and Sergeev A V 1996 Int. J. Mod. Phys. B 10635

[15] Manninen A, Suoknuuti J, Leivo M and Pekola J 1999 Appl. Phys. Lett. 743020 